\title{
Tuning the perpendicular magnetic anisotropy in Co/Pt multilayers grown by facing target sputtering and conventional sputtering
}

\author{
Oğuz Yıldırım ${ }^{\mathrm{a}, *}$, Miguel A. Marioni ${ }^{\mathrm{a}, 1}$, Claudiu V. Falub ${ }^{\mathrm{b}}$, Hartmut Rohrmann ${ }^{\mathrm{b}}$, \\ Dominik Jaeger ${ }^{\mathrm{b}}$, Marco Rechsteiner ${ }^{\mathrm{b}}$, Daniel Schneider ${ }^{\mathrm{b}}$, Hans J. Hug ${ }^{\mathrm{a}, \mathrm{c}}$ \\ ${ }^{a}$ Empa, Swiss Federal Laboratories for Materials Science and Technology, Laboratory for Magnetic and Functional Thin Films CH-8600 Dübendorf, \\ Switzerland \\ ${ }^{\mathrm{b}}$ Evatec AG, Trübbach $\mathrm{CH}-9477$, Switzerland \\ ${ }^{\mathrm{C}}$ Department of Physics, University of Basel, Basel CH-4056, Switzerland
}

\section{A R T I C L E I N F O}

\section{Article history:}

Received 16 July 2021

Accepted 10 September 2021

PACS:

pacs

\begin{abstract}
A B S T R A C T
Magnetic properties of $[\mathrm{Co}(0.4 \mathrm{~nm}) / \mathrm{Pt}(0.7 \mathrm{~nm})]_{5}$ multilayers are tailored by controlling the sputtering voltage during growth of cobalt layers by a facing target cathode. It is shown that increasing sputtering voltage up to $150 \mathrm{~V}$ leads to an improved crystalline texture and this results in larger magnetic anisotropies together with increased out-of-plane coercive fields. At a higher cathode voltage of $540 \mathrm{~V}$, however, crystalline texture quality slightly worsens and this is accompanied by a decrease in the effective anisotropy. Using facing target cathode sputtering, the crystalline structure of the multilayers can be controlled without applying any heat treatment and this can be utilized to optimize the magnetic properties of $\mathrm{Co} / \mathrm{Pt}$ multilayers for specific applications.
\end{abstract}

(c) 2021 The Author(s). Published by Elsevier Ltd on behalf of Acta Materialia Inc. This is an open access article under the CC BY license (http://creativecommons.org/licenses/by/4.0/)
Magnetic multilayers yielding perpendicular magnetic anisotropy (PMA) are key components for various applications such as magnetoresistive field sensors or magnetic memory devices. Magnetic tunnel junctions (MTJs) for instance are used in both sensor and memory applications. As already shown in previous studies, MTJ-based devices with PMA show substantially higher bit density, high thermal stability and require lower current densities as compared to the devices with in-plane anisotropy. $[1,2]$ Conventionally, PMA in a magnetic multilayer system is controlled either by changing the layer thickness or through modifying the interface qualities. If the layer thickness is kept fixed in a magnetic multilayer, heat treatment can be applied to modify the PMA. Annealing magnetic multilayers under certain conditions improves the crystalline structure providing sharper interfaces which in turn results in larger PMA. [3] Heat treatment on these multilayers has to be performed with a great care as the excessive temperatures can cause unwanted effects and deteriorate the PMA. [4] Alternatively, the quality of the interfaces and thus the PMA can be tuned by regulating the deposition pressure during sputtering. The deposition pressure affects the mean free path of

\footnotetext{
* Corresponding author::

E-mail address: oguz.yildirim@empa.ch (O. Yıldırım).

1 Deceased
}

the sputtered atoms and alters the impact of the bombardment with accelerated atoms on the growing film. [5,6]

In a recent work, we have demonstrated that the crystallinity of Pt films on sapphire substrates can be tuned from a disordered nanocrystalline state to a highly epitaxial films via controlling the thermal energy provided on the subtrates when these films were grown by facing target cathode (FTC) sputtering. [7] Moreover, we have also shown that the facing target sputtering source provides lower adatom energies, comparable to thermal energies, i.e. much lower than those typically occurring when sputtering with a conventional on-axis magnetron source. In our previous work we have shown that the low energies possible with FTC sputtering can substantially change the crystallinity of a Pt layer grown on single crystalline sapphire substrates.

In this work, $[\mathrm{Co}(0.4 \mathrm{~nm}) / \mathrm{Pt}(0.7 \mathrm{~nm})]_{5}$ multilayers are deposited by a facing target cathode and a conventional magnetron sputtering gun for Co and Pt layers, respectively. The dependence of magnetic and structural properties on the facing target voltage is investigated. Different cathode voltages on the FTC are obtained by combining DC and RF powers simultaneously. The parameters of the conventional sputtering source used for the Pt remained fixed for all of the samples. $[\mathrm{Co}(0.4 \mathrm{~nm}) / \mathrm{Pt}(0.7 \mathrm{~nm})]_{5}$ multilayers were grown on thermally oxidized Si substrates and $10 \mathrm{~nm}$ thick Pt seed layers. These multilayers are also capped with a $2 \mathrm{~nm}$ thick Pt layer to prevent from oxidation. In order to control the energy of the 
a)

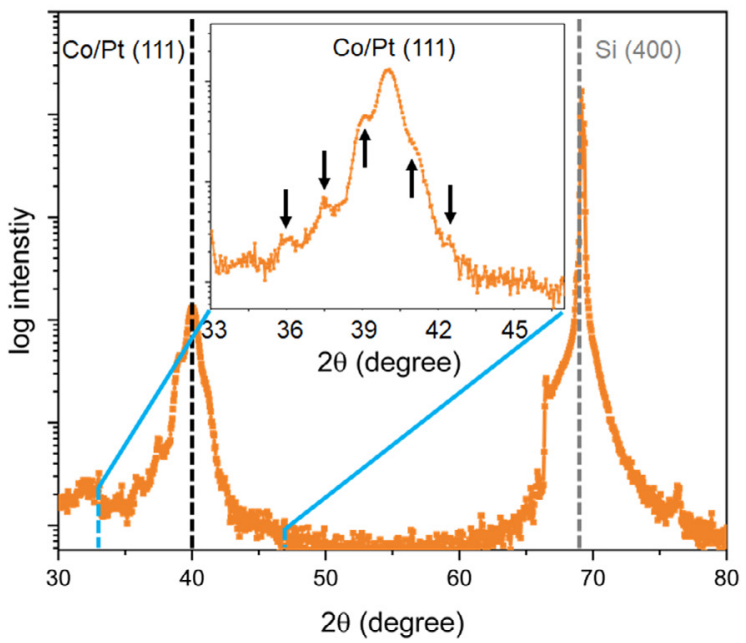

b)

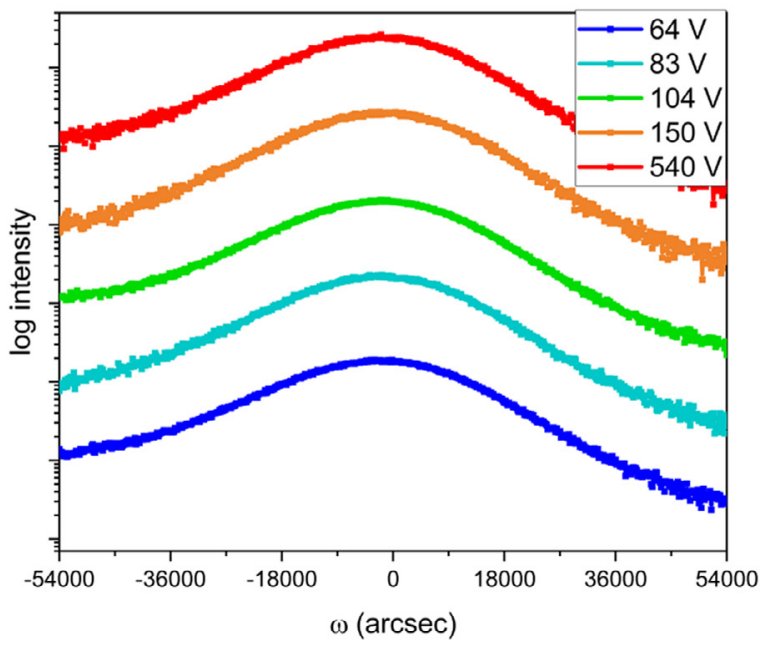

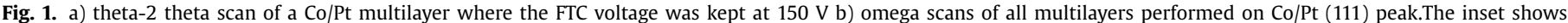
an enlarged view of the theta-2-theta data for 2-theta ranging from 33 to 47 degrees.

Table 1

Deposition voltages and powers together rocking curve full-width at half-maximum values.

\begin{tabular}{llll}
\hline $\begin{array}{l}\text { FTC Voltage } \\
(\mathrm{V})\end{array}$ & $\begin{array}{l}\text { DC power } \\
(\mathrm{W})\end{array}$ & $\begin{array}{l}\text { RF power } \\
(\mathrm{W})\end{array}$ & $\begin{array}{l}\text { RC FWHM (deg) } \\
\left( \pm 0.09^{\circ}\right)\end{array}$ \\
\hline 64 & 20 & 240 & 8.30 \\
83 & 20 & 480 & 8.21 \\
104 & 120 & 300 & 7.96 \\
150 & 120 & 500 & 7.87 \\
540 & 120 & - & 8.02 \\
\hline
\end{tabular}

sputtered species impacting on the Co layers during their growth, different combinations of $\mathrm{DC}$ and RF powers were applied to the FTC (table 1). It is worth noting that we have also grown multilayers where both Co and Pt were deposited by FTC. However, these multilayers did not show PMA, which we attribute to the amorphous structure of the Pt layers arising from the FTC deposition [7] and therefore results in rough interfaces and only in-plane anisotropies. The structural and magnetic properties of the multilayers were investigated by X-ray diffraction (XRD) and magnetometry. XRD measurements were recorded on a Bruker D8 Discover equipped with a $\mathrm{Cu}-K_{\alpha}$ radiation source. Magnetometry measurements were performed on a custom design polar Kerr magnetometer and on a vibrating sample magnetometer (VSM) attached to a physical properties measurement system (PPMS) from Quantum Design.

Shown in Fig. 1 a) is theta - 2 theta scan of a sample grown at FTC voltage of $150 \mathrm{~V}$. Theta - 2 theta scans are performed for all of the samples, however the data obtained for each multilayer showed only negligible differences. Therefore, for simplicity, data obtained from a single sample is shown. XRD scans revealed only the Co/Pt (111) superlattice peak and the substrate peak (1 fig. [a]) for all samples. Co/Pt multilayers grown on the Pt (111) seed have a crystalline texture aligned in (111) direction and the diffraction peak is located at angles close to $\mathrm{Pt}(111)$ position $\left(39.75^{\circ} \mathrm{C}\right)$ [8], as commonly observed for Co/Pt multilayers. [9] Shown in the inset is the zoomed-in view of the XRD pattern given in Fig. 1 a). Superlattice satellite peaks are indicated with arrows and confirm the layered nature of the samples. Rocking curve (RC) measurements performed by aligning on the $\mathrm{Co} / \mathrm{Pt}$ (111) superstructure peak are shown in Fig. 1 (b). Full-width at half-maximum (FWHM) values of RCs are obtained by fitting the RCs with Gaussian functions and given in table 1 . Note that FWHM values are larger compared to the single crystalline systems but comparable to the values reported for similar sputter-deposited multilayer systems. [10] The FWHM values obtained from the (111) diffraction peak initially decreases with increasing FTC voltage starting from the lowest value. The lowest FWHM value is obtained from the multilayer where FTC voltage was kept at $150 \mathrm{~V}$. Higher than $150 \mathrm{~V}$, FWHM increases slightly. It is worth noting here that due to the limitations from our electronics, we were not able sustain a plasma for voltages between $150 \mathrm{~V}$ and $540 \mathrm{~V}$ on the FTC source. Therefore, this voltage range remains unexplored. Nevertheless, the different FWHM values of the omega scans indicates a change in the crystal structure. More specifically, films grown at higher FTC voltages showed lower FWHM values thus improved crystalline texture.

Fig. 2 shows the out-of-plane a) and in-plane b) $M\left(\mu_{0} H\right)$-loops measured by polar Kerr and VSM, respectively, obtained from the multilayers. The $100 \%$ remanence visible in a), as well as the hardaxis loop behavior in $b$ ) reveals that all samples have sufficient PMA to overcome the films magnetostatic energy, i.e. $K_{\text {eff }}>0$ for all samples. All multilayers measured by using both VSM and polar-Kerr magnetometer. VSM measurements were performed for in- and out-of-plane directions, while polar-Kerr magnetometry, by definition, was only performed for the out-of-plane direction. From the VSM measurements, saturation magnetization for all the samples was found to be $1.35( \pm 0.1) \mathrm{MA} / \mathrm{m}$. VSM measurements are not as sensitive as Kerr magnetometry to the very small changes in magnetic fields. Therefore, out-of-plane hysteresis curves obtained from a polar-Kerr magnetometer are shown in Fig. 2 a), which enables more precise assessment of coercive field values. Multilayers grown with different FTC voltage for Co layers saturate at different magnetic fields for in-plane measurements. Namely, from lowest FTC voltage of $64 \mathrm{~V}$ to $150 \mathrm{~V}$ coercive field increases. For the sample grown with FTC voltage of $540 \mathrm{~V}$, coercive field drops slightly as compared to the sample grown with $150 \mathrm{~V}$ FTC voltage.

In Fig. 3 a) shown is the $H_{\mathrm{c}}$ and effective anisotropy $\left(K_{\text {eff }}\right)$ values in dependence of the FTC voltage. $H_{c}$ values were extracted from out-of-plane polar Kerr loops that are shown in Fig. 2 a) and they were found to be very comparable to a previous study. [11] $K_{\text {eff }}$ values were obtained from in-plane magnetization curves. [14] Both $H_{\mathrm{c}}$ and $K_{\text {eff }}$ increases with increasing FTC voltage from $64 \mathrm{~V}$ to 150 $\mathrm{V}$ during Co layer growth. However, at a higher FTC voltage of 540 $\mathrm{V} H_{\mathrm{c}}$ and $K_{\text {eff }}$ decreases. A similar change in effective anisotropy in relation to the annealing temperature was previously reported by 
a)

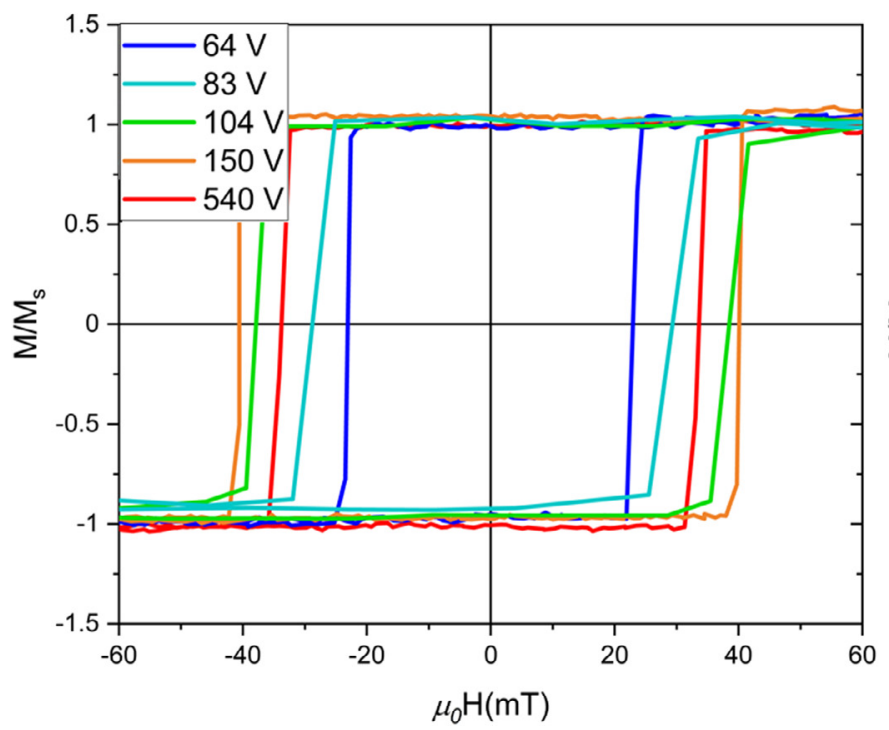

b)

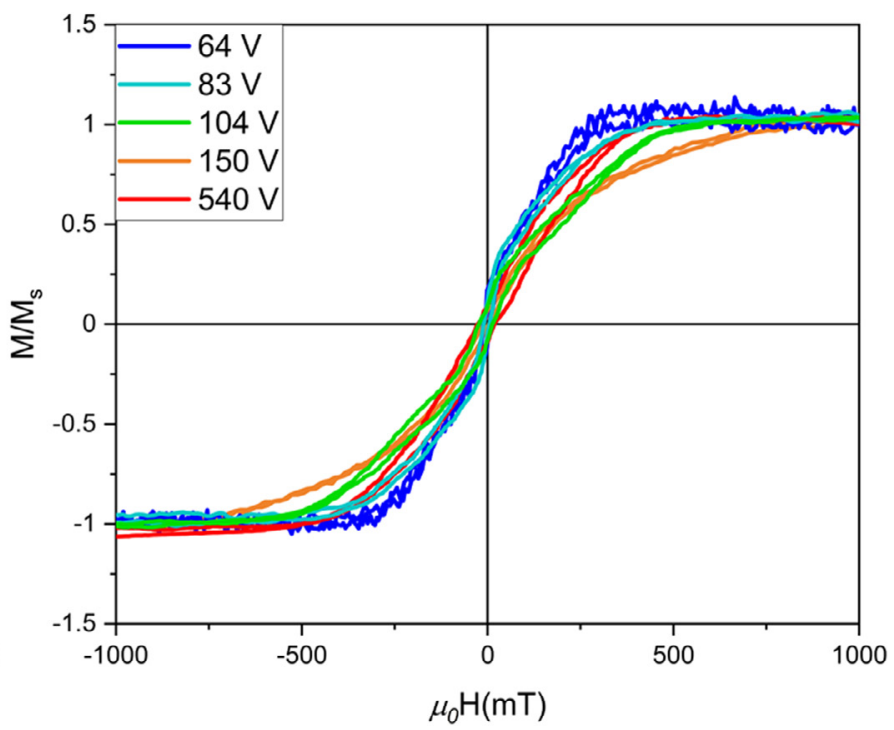

Fig. 2. a) Out-of-plane and b) in-plane magnetometry data obtained from Co/Pt multilayers.

a)

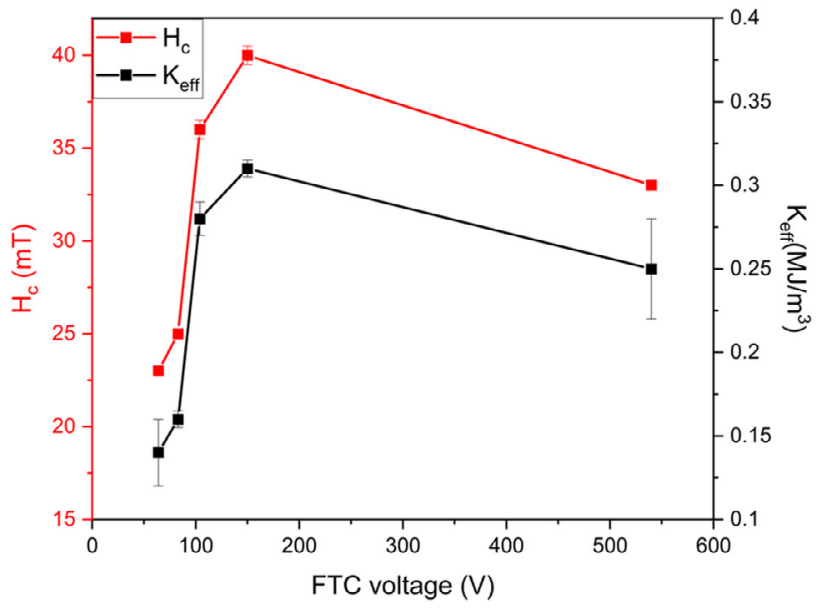

b)

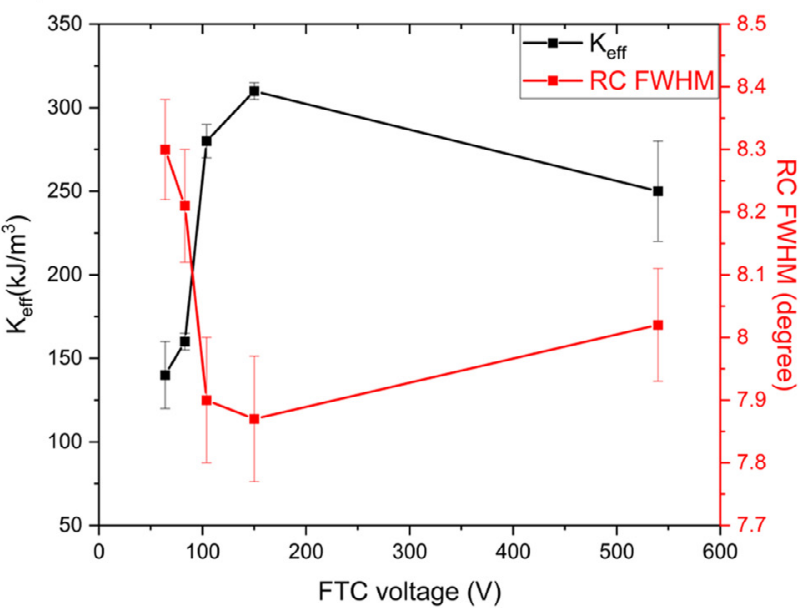

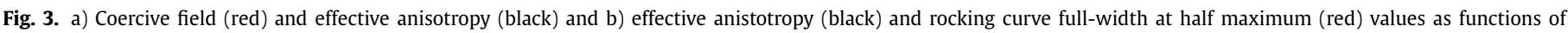
FTC voltage.

Lee et al. [15] For a comparable cobalt thickness, they show that $K_{\text {eff }}$ increases up to annealing temperature of $400{ }^{\circ} \mathrm{C}$ and at higher annealing temperatures $K_{\text {eff }}$ drops. RC FWHM and $K_{\text {eff }}$ values are shown together on Fig. 3 b), where a clear correlation can be observed between the texture and anisotropy. Namely, increasing FTC voltage up to $150 \mathrm{~V}$ leads to narrower RCs and larger $K_{\text {eff }}$ and a very small change occur when the voltage is raised to $540 \mathrm{~V}$.

In Co/Pt multilayers one of the main drivers for the magnetic anisotropy is the interface quality. $[12,13,16]$ Improved (111) texture of the magnetic multilayers results in sharper interfaces and thus larger PMA. [17] A crystalline texture in these magnetic multilayers are often induced by a proper choice of a crystalline seed layer. [3] Pt is one of the most common materials used as a seed layer in such multilayers. Another parameter that plays an important role in the establishment of the crystalline texture is the adatom energy. In a sputtered system, a good (111) texture often requires sufficiently high adatom energies while the low adatom energies during sputter deposition results in lower structural order. [7] On the other hand, very high adatom energies and ion/neutral bombardment also causes unwanted effects such as interfacial rough- ening and intermixing of multilayers which deteriorates the PMA. [4] Here we show that increasing cathode voltage increases the adatom energy and this is reflected as an improved texture. At a cathode voltage higher than a certain level, adatom energy becomes too high and start inducing defects at the interface. In order to avoid such defects occurring at the interfaces during deposition of these multilayer systems by conventional on-axis sputtering, deposition power and pressure are kept at the lowest possible limits. At this point, FTC deposition has an advantage over on-axis magnetron sputtering as it provides lower adatom energies due to its deposition geometry. Achieving such low adatom energies allows for lower defect formation and better control over the interfaces. [18]

Observed dependency of the crystalline texture to the cathode voltage is similar to the changes induced by post-deposition annealing. $[15,19]$ It is already shown that the texture and the magnetic anisotropy of the Co/Pt multilayers can be improved by applying a post-deposition annealing up to intermediate temperatures of around $200{ }^{\circ} \mathrm{C}$ to $400{ }^{\circ} \mathrm{C}$. [3,19] Above such an optimum temperature, interfacial mixing starts and therefore the magnetic 
anisotropy decays as a result of interfacial mixing. In this study, deposition parameters for the Pt layers were kept the same. Therefore, all of the structural changes were attributed to the change in the sputtering voltage during Co deposition. Accordingly, it is shown here that tuning FTC voltage modifies the adatom energy at the level of thermal energies and provides a finer control over structural and magnetic properties.

$[\mathrm{Co}(0.4 \mathrm{~nm}) / \mathrm{Pt}(0.7 \mathrm{~nm})]_{5}$ multilayers with PMA are grown by combining conventional magnetron sputtering and FTC sputtering. It is shown that the larger anisotropies and larger out of plane coercivities in these multilayers can be obtained at FTC voltages between $150 \mathrm{~V}$ and $540 \mathrm{~V}$ during cobalt deposition. Tunability of the adatom energies achieved by FTC deposition can be beneficial for different applications where fine tailoring of the magnetic properties required and heat treatment cannot be applied.

\section{Declaration of Competing Interest}

The authors declare that they have no known competing financial interests or personal relationships that could have appeared to influence the work reported in this paper.

\section{Acknowledgments}

We dedicate this study to a very good scientist, mentor, colleague and friend; Miguel A. Marioni, he may rest in peace. We are grateful to Marco Padrun and Maurus Tschirky for the continued support. We would like to acknowledge financial support from the Swiss Federal Commission for Technology and Innovation (CTIProject Nr. 18940.2 PFNM-NM).

\section{References}

[1] S. Mangin, D. Ravelosona, J.A. Katine, M.J. Carey, B.D. Terris, E.E. Fullerton, Nature Mater. 5 (2006) 210-215.

[2] S. Ikeda, K. Miura, H. Yamamoto, K. Mizunuma, H.D. Gan, M. Endo, S. Kanai, J. Hayakawa, F. Matsukura, H. Ohno, Nature Mat. 9 (2010) 721-724.

[3] J. Chatterjee, T. Tahmasebi, S. Mertens, G.S. Kar, T. Min, J. De Boeck, IEEE T. Magn. 50 (2014) 11.

[4] P.F. Carcia, S.I. Shah, Appl. Phys. Lett. 56 (1990) 2345.

[5] G.A. Bertero, R. Sinclair, J. Magn. Magn. Mater. 134 (1994) 173-184.

[6] S.M. Rossnagel, J. Vac. Sci. Technol. A 7 (1989) 1025.

[7] O. Yildirim, A. Borzi, C. Falub, H. Rohrmann, D. Jaeger, M. Rechstainer, D. Schneider, A. Neels, H.J. Hug, M.A. Marioni, Scr. Mater. 194 (2021) 113689.

[8] J.W. Arblaster, Platinum Metal. Rev. 41 (1) (1997) 12.

[9] O. Yildirim, D. Hilliard, S.S.P.K. Arekapudi, C. Fowley, H. Cansever, L. Koch, L. Ramasubramanian, S. Zhou, R. Boettger, J. Lindner, J. Fassbender, O. Hellwig, A. Deac, ACS Appl. Mater. Interfaces 12 (2020) 9858-9864.

[10] D. Weller, L. Folks, M. Best, E.E. Fullerton, B.D. Terris, G.J. Kusinski, K.M. Krishnan, G. Thomas, J. Appl. Phys. 89 (2001) 11.

[11] P. Chowdhury, P.D. Kulkarni, M. Krishnan, H.C. Barshilia, A. Sagdeo, S.K. Rai, G.S. Lodha, D.V.S. Rao, J. Appl. Phys. 112 (2012) 023912.

[12] S.S.P. Parkin, R.F.C. Farrow, R.F. Marks, A. Cebollada, G.R. Harp, R.J. Savoy, Phys. Rev. Lett. 72 (1994) 3718.

[13] R.F.C. Farrow, G.R. Harp, R.E. Marks, T.A. Rabedau, M.F. Toney, D. Weller, S.S.P. Parkin, J. Cry. Growth 133 (1993) 47.

[14] M.T. Johnson, P.J.H. Bloemen, F.J.A.d. Broeder, J.J. de Vries, Rep. Prog. Phys. 59 (1996) 1409-1458.

[15] Y. Lee, D.S. Son, S.H. Lim, S.R. Lee, J. Appl. Phys. 113 (2013) 216102

[16] C.-J. Lin, G.L. Gorman, C.H. Lee, R.F.C. Farrow, E.E. Marinero, H.V. Do, H. Notarys, C.J. Chien, J. Magn. Magn. Mat. 93 (1991) 194-206.

[17] G.A. Bertero, R. Sinclair, J. Magn. Magn. Mater. 134 (1994) 173-184.

[18] H.-K. Kim, S.W. Kim, Appl. Phys. Lett. 88 (2006) 083513.

[19] S. Sumi, Y. Kusumoto, Y. Teragaki, K. Torazawa, J. Appl. Phys. 73 (1993) 6835 